\title{
Aprovechamiento de Patas de Pollos como Alternativa para disminuir Residuos Generados en los Mataderos
}

\author{
Poliana F. Almeida ${ }^{(1,2)}$, José A. A. Salles ${ }^{(1)}$, Thiago M. B. Farias ${ }^{(1)}$, José C. Curvelo Santana ${ }^{\left(1^{\star}\right)}$ \\ (1) Programa de Mestrado em Engenharia de Produção, Universidade Nove de Julho (UNINOVE), \\ Av. Francisco Matarazzo, 612, CEP: 05001-100, São Paulo-Brasil. (e-mail: jccurvelo@uninove.br) \\ (2) Instituto Federal de Educación, Ciencia y Tecnología Mato Grosso, IFMT-Campus São Vicente, \\ MT, Brasil. (e-mail poliana.fernandes@hotmail.com)
}

Recibido Oct. 26, 2011; Aceptado Dic. 20, 2011; Versión final recibida Feb. 06, 2012

\section{Resumen}

Se presenta un estudio sobre la producción de jaleas y gelatinas a partir de las patas de pollo, usualmente producidas como desechos de los mataderos de aves. La investigación se llevó a cabo en dos etapas. En la primera etapa, las jaleas se obtuvieron a partir de las patas del pollo y se evaluaron sus cualidades sensoriales para verificar su aceptabilidad. En la segunda etapa, se produjo gelatinas y se analizó también sus cualidades sensoriales para comprobar su aceptabilidad. Los resultados mostraron que la mayoría de los catadores aprobaron los productos con más de 7 puntos en la escala hedónica y más del 80\% dijeron que consumirían los productos. Los niveles de proteínas en las jaleas y gelatinas de patas de pollo superaron en cuatro veces los niveles de los productos comerciales, lo que indica una superioridad con respecto a las calidades químicas y potencialmente medicinales.

\section{Use of Chicken Feet as an Alternative to the reduce Wastes from Slaughterhouse}

\begin{abstract}
A study on the production of jelly and gelatin from chicken feet, produced as wasted from chicken slaughterhouse is presented. The study was conducted in two parts. In the first part, jellies were produced from chicken feet and sensory quality was done to verify their acceptability. In the second step, gelatins were elaborated from chicken feet and also subjected to sensory analysis. Results showed that most experts in the panel accepted the products with more than 7 points in the hedonic scale and $80 \%$ said that they would consume these chicken products. Gelatin and jelly protein contents were four times larger than commercial products, indicating superiority in their chemistry and potential medicinal qualities.
\end{abstract}

Keywords: chicken feet, sensorial analysis, food qualities, gelatins, jelly 


\section{INTRODUCCIÓN}

La reducción de los impactos ambientales causados por la eliminación de residuos industriales favorece la preservación de los componentes del agua y del suelo en todo el mundo. Diversas investigaciones muestran el aprovechamiento de agua de los efluentes residenciales para la irrigación de diferentes cultivos agrícolas (Coria y Vegas, 2011; Passarini et al., 2012; Rodríguez et al., 2008). Los mataderos generan efluentes líquidos compuestos de grasas, proteínas y restos de animales. También generan los desechos sólidos, tales como: los huesos, las entrañas, patas, cabezas, piel y otros restos de carcasas. Comúnmente, gran parte de estos desechos no son utilizadas por estas empresas, a menudo son descartadas en el vertederos (Biazus et al., 2006). Es importante mencionar que, en determinados lugares los residuos animales son considerados como materia prima y en otros lugares son utilizados como productos tradicionales de mayor valor agregado (Alvís et al., 2009a y 2009b; Aquino et al., 2009; Castillo et al., 2009; Hernández et al., 2009; Machado et al., 2010; Michalewicz et al., 2011). En este contexto, se hace necesario buscar alternativas de aprovechamiento y reutilización de los residuos obtenidos en los mataderos.

En Brasil, la industria considera a las extremidades del pollo (patas) como un subproducto de escaso interés comercial. Los precios por este subproducto son muy bajos, debido a que la población no tiene interés en consumirlo. También, gran parte de este producto es desechado como un residuo, por falta de un mercado y otra parte es utilizado en la fabricación de los alimentos para animales (Rivera et al., 2000). Este comportamiento está cambiando. Era común considerar como inútil a los desechos de alimentos que contienen varias importantes substancias importantes y que, ahora se convierten en productos de amplia aceptación comercial. De acuerdo con Gómez-Guillén et al. (2011) y Karim y Bhat (2009) una alternativa a la valorización de residuos es el desarrollo de nuevos productos dando a los residuos un destino más noble y de mayor valor comercial. Liu et al. (2001) estudiaron el uso de los tarsos de las aves para la preparación de la gelatina comestible. En este estudió fue encontrado que, cuando el colágeno se somete a un tratamiento térmico en una atmósfera húmeda y parcialmente hidrolizada se convierte en gelatina. Las entrañas de las aves han sido utilizadas para hacer harina por Costa et al. (2008). De esta manera, han contribuido con la auto sustentabilidad de regiones donde se instalan los mataderos.

El colágeno es una proteína fibrosa que tiene numerosas aplicaciones principalmente en la industria farmacéutica y alimenticia. Esto es debido a sus propiedades físico-químicas y tienen la habilidad de formar geles térmicamente reversibles. También puede ser usado como agente emulsificante, estabilizante, o para mejorar algunas características como textura y capacidad de retención de agua (Saxena et al., 2009; Serna-Cock et al., 2010). La gran mayoría de los colágenos comerciales son procedentes de mamíferos y son obtenidos principalmente de la piel del cerdo y del ganado. Mas, por razones socio-culturales, existe una creciente demanda de fuentes alternativas. También por razones socio-culturales e religiosos, productos de la piel del cerdo no son aceptables para el judaísmo y el Islam y los productos de carne de res son aceptables sólo si se ha tramitado de acuerdo con los requisitos religiosos (Giménes et al., 2009; Gómes-Gulién et al., 2011; Karim and Bhat, 2009). Adicionalmente, ocurre que con frecuencia la encefalopatía espongiforme bovina (EEB) y diversas enfermedades en la boca. Estos han sido serios problemas para la salud humana y por lo tanto los subproductos de los mamíferos están limitados a la utilidad de procesamiento de alimentos funcionales, productos cosméticos y farmacéuticos (Gómes-Gulién et al., 2011; Karim y Bhat, 2009). Ante esta problemática, diversas investigaciones muestran otras alternativas de extracción de colágeno a partir de fuentes marinas, como por ejemplo: del esturión (Acipenser baeri) (Hao et al., 2009); de la raya (Raja kenojei) (Gómez-Guillén et al., 2011); del calamar gigante (Dosidicus gigas) (Giménez et al., 2009); de salmón del Atlántico (Salmo salar) (Arnesen y Gildberg, 2007); de la corvina sin (Johnius dussumieri); del marrajo jurel (Decapterus macrosoma) (Cheow et al., 2007); del bagre de canal (Ictalurus punctatus) (Yan et al., 2007) y otros peces (Gómez-Guillén et al., 2011; Karim y Bhat, 2009).

Es importante destacar que, durante el proceso de raspado de piel de vacuno utilizado en la producción de colágeno, se producen residuos que contienen cromo. Este producto generalmente 
es descartado y puede contaminar el medio ambiente (Gómez-Guillén et al., 2011). Adicionalmente, el precio del kilogramo de piel de cerdo para la fabricación de colágeno está alrededor de U\$ 0,55 (dólar americano) y el valor de la materia prima de ganado es de U\$ 0.88 (dólar americano) por kilogramo. En cuanto que, el caso específico de las patas de pollo, las cuestiones culturales, gastronómicas y las tradicionales (superstición), son factores que justifican el desprecio por el producto. Estas características del mercado nacional son cruciales para definir sus valores de venta bajos. Siendo un precio alrededor de U\$ 0.588/tonelada (MFRURAL, 2010). En este contexto, la búsqueda de fuentes alternativas para obtener colágeno es también un problema ambiental. Por lo tanto, las fuentes de colágeno de las especies porcina y bovina son más caras que la fuente de pollo. Finalmente, este trabajo tuvo como objetivo obtener jaleas y gelatinas a partir de patas de pollo y su caracterización de acuerdo a sus cualidades sensoriales.

\section{MATERIALES Y MÉTODOS}

La investigación se divide en dos Etapas. Al principio tratamos de ajustar la formulación, la metodología de procesamiento y su aceptabilidad por análisis sensorial. Por lo tanto se propuso la producción de dos sabores distintos y se compararon con el análisis sensorial. Después, la investigación fue diseñada para probar una gelatina experimental por adición de otro sabor y la eliminación de algunos ingredientes. Comparándola con una marca consolidada en el mercado. Como materia prima para la elaboración de gelatina se utilizaron patas de pollos recién sacrificados en el Instituto Federal de Educación, Ciencia e Tecnología Mato Grosso - IFMT Campus São Vicente.

En la primera etapa. Fueron lavadas las patas. Después fueron retiradas las uñas y luego se lavaron de nuevo las patas con agua fría para eliminar cualquier residuo de suciedad. Posteriormente fueron sometidos al proceso de cocción a $120^{\circ} \mathrm{C}$ durante 20 minutos. Después de la cocción, la porción líquida resultante se separó, se filtró y la muestra se colocó en un recipiente de vidrio. La muestra fue sometida a refrigeración y después de solidificado la grasa que se acumuló en la superficie debido a la menor densidad, favoreció su separación y posterior eliminación. Finalmente, una mezcla de colágeno y $500 \mathrm{~mL}$ de agua fueran colocados en un recipiente de acero inoxidable y fue calentado con $200 \mathrm{~g}$ de azúcar y aditivos. Inicialmente, fueron preparadas dos muestras jaleas llamadas GP y GCB. La muestra GP se formuló con el sabor de la piña y la muestra GCB con el sabor de chocolate blanco. En la muestra GP fue agregado colorante amarillo y esencia de piña, ácido ascórbico y ácido cítrico. En la muestra GCB se añadió ácido cítrico y polvo Siber sabor a chocolate blanco. En ambas muestras se agregó leche entera en polvo y la pectina, con el objetivo de mejorar la consistencia final.

En la segunda etapa del experimento, las patas limpias fueron sometidas al proceso de cocción a $60^{\circ} \mathrm{C}$ durante $8 \mathrm{~h}$. Después de la cocción, la porción líquida resultante fue separada de las grasas en un filtró con algodón y fue secada en estufa con circulación de aire. La muestra secada se colocó en un recipiente de vidrio. Se añadieron $500 \mathrm{~mL}$ de agua y se produjo una muestra de gelatina llamada GU, formulado con sabor a uva. A la muestra $\mathrm{GU}$ se añadieron $0,5 \mathrm{~g}$ de conservante sorbato de potasio y $10 \mathrm{~g}$ de polvo Siber sabor a uva. Este tiene en su composición colorantes, almidón, acidulante, regulador de la acidez y anti-humectante. Las gelatinas comerciales utilizadas fueron de la marca Dr. Oetker sabor a uva (GUC) producida de acuerdo con su fabricante (Arnesen y Gildberg, 2007; Karim y Bhat, 2009; Liu et al., 2001; Yan et al., 2007). Las análisis de la composición de la gelatina fueron hachas de acuerdo con Arnesen y Gildberg (2007); Cheow et al. (2007); Hao et al. (2009) y Karim y Bhat (2009).

La evaluación sensorial fue realizada por 30 catadores no entrenados. Todos fueron estudiantes del campus de São Vicente del IFTMA. El equipo incluyó a individuos de ambos sexos, con edades comprendidas entre 15 y 25 años. Las muestras fueron servidas de forma monódica, en cabinas individuales en vasos de plástico desechables codificados con cantidades estandarizadas. Los catadores tenían a su disposición un vaso de agua que se utiliza para enjuagar la boca entre las muestras y las respuestas fueron registradas en una tabla de respuestas estandarizada. Las pruebas afectivas se utilizan cuando se necesita conocer el "estado afectivo" de los consumidores por el producto, y para ello se utilizan escalas hedónicas. De los valores relativos de aceptación se 
puede inferir la preferencia. Es decir, las muestras más aceptadas son las más preferidas. Así, se evaluaron los atributos de color, sabor, aroma, textura e incluso la disponibilidad de consumir el producto. Se utilizó la prueba con la escala afectiva hedónica de 9 puntos, que van desde 1-no les gusta muchísimo a 9-les gustó muchísimo. Para los atributos apariencia, aroma, sabor y textura; y la prueba para determinar la predisposición al consumo con una escala de 1- no consumir a 6consumir todos los días. Con los resultados obtenidos de los cuestionarios se tabularon las respuestas en orden de frecuencia. El método de $t$ Student fue utilizado para el análisis estadísticas de las muestras (Alvis et al., 2008 y 2011; Costa et al., 2008; Ronceros et al., 2008; Rodrígues y Gualdron, 2011; Santana et al., 2010).

\section{RESULTADOS Y DISCUSIÓN}

Después de la extracción, fue obtenido un rendimiento de $6,5 \%$ de masa seca de jalea, con relación a la masa de las patas de pollo. La Tabla 1 muestra los valores promedios de las analices sensoriales de las jaleas de patas de pollo obtenidas en la primera etapa de la investigación. Se observa que los resultados de todas las cualidades sensoriales recibieron altas calificaciones en sus evaluaciones. El más bajo estaba cerca de los 7 puntos en la escala hedónica (que corresponden a: me gustaba), mientras que la mayoría recibió calificaciones cerca de 8 puntos (me gustó mucho), lo que indica que no hubo diferencias significativas entre las muestras y que las dos jaleáis fueron bien aceptados por los consumidores. 74\% y $85 \%$ de los catadores están dispuestos a consumir una o más veces durante la semana, jalea de piña y chocolate blanco, respectivamente; lo que confirma la aceptación de estos productos. En general, los resultados indicaron que los catadores de las muestras GAB y GCB y han gustado mucho o muchísimo y que el $50 \%$ de ellos os consumirían más de una vez por semana. El análisis estadística utilizando lo teste de $t$ Student mostró que no hubo diferencias significativas entre las muestras de las cualidades, aroma, sabor y textura. Sin embargo, diferencias significativas fueran observadas entre las apariencias de las muestras, para el nivel de confidencia de $95 \%$, con mejores puntuaciones para la jalea comercial, aunque no se descalifica la jalea de patas de pollo, ya que su valor excede la escala de "me gustaba". La Fig. 1 muestra los valores sensoriales en escala hedónica (Alvis et al., 2008; Costa et al., 2008; Santana et al., 2010; Silva et al., 2010).

Tabla 1: Evaluación de las cualidades sensoriales de las jaleas de las patas de pollo. En la Tabla, $\mathrm{GP}=$ Piña y $\mathrm{GCB}=$ Chocolate Blanco; $\mathrm{c}, \mathrm{d}=$ =Letras distintas indican diferencias significativas entre las muestras

\begin{tabular}{|c|c|c|c|c|c|}
\hline \multirow{2}{*}{ Muestras } & \multicolumn{5}{|c|}{ Valores sensoriales promedios } \\
\cline { 2 - 6 } & Aroma & Apariencia & Sabor & Textura & Deseo de consumir (\%) \\
\hline GP & $7,6^{\mathrm{a}}$ & $7,8^{\mathrm{b}}$ & $7,9^{\mathrm{d}}$ & $7,9^{\mathrm{e}}$ & 74 \\
\hline GCB & $7,5^{\mathrm{a}}$ & $6,8^{\mathrm{c}}$ & $7,7^{\mathrm{d}}$ & $8,0^{\mathrm{e}}$ & 85 \\
\hline
\end{tabular}

La Tabla 2 muestra los valores medios del análisis sensorial de la gelatina de patas de pollo y de la muestra comercial. Los promedios obtenidos por la gelatina comercial fueron cerca o superiores a 8 para todas las calidades sensoriales, lo que indica que la mayoría de los catadores dijeron haber gustado mucho que de la gelatina. En cuanto a la gelatina de tarsos de pollo los valores fueron cerca de 7, lo que indica que la mayoría de los catadores, dijo que "le gustaba" esta gelatina. El análisis estadístico utilizando lo teste de $t$ Student mostró que no hubo diferencias significativas entre las muestras de las cualidades aroma, apariencia y sabor. Sin embargo, diferencias significativas entre las texturas de las muestras fueron observadas, con mejores puntuaciones para la gelatina comercial, aunque no se descalifica la gelatina de tarsos de pollo, ya que su valor excede la escala de "gustó moderadamente". La pequeña diferencia en la textura se debe al hecho de que la gelatina experimental de uva presenta como más consistente que los productos disponibles en el mercado, haciendo que algunos productos extraños a los consumidores acostumbrados a menor consistencia (Alvis et al., 2008; Costa et al., 2008; Santana et al., 2010; Silva et al., 2010). Esto puede ser probado por la cantidad de agua presente en las muestras, ya que una mayor cantidad de agua en la composición hace que el producto sea menos duro que otros. Sin embargo, estas diferencias en la evaluación de la gelatina no causa impacto 
negativo en los atributos sensoriales evaluados en las pruebas en ambas muestras. Para una evaluación del deseo de consumir las gelatinas en la prueba sensorial, se observa que los valores asignados por los evaluadores para las intenciones del consumo de GUC fueron ligeramente superiores. Sin embargo, más del $83 \%$ de los catadores dijo que tienen intenciones de consumir gelatina obtenida de patas de pollo (GU), indicando que esto también puede ser objeto de comercio, ya que su aceptación fue alta (Alvis et al., 2008; Costa et al., 2008; Santana et al., 2010; Silva et al., 2010). Después de la extracción y secado, fue obtenido un rendimiento de $5,4 \%$ de masa de gelatina, con relación a la masa de las patas de pollo. Que es de 78,5\% de proteínas, $9,7 \%$ de humedad, 6,9 de grasas y $4,85 \%$ de cenizas.

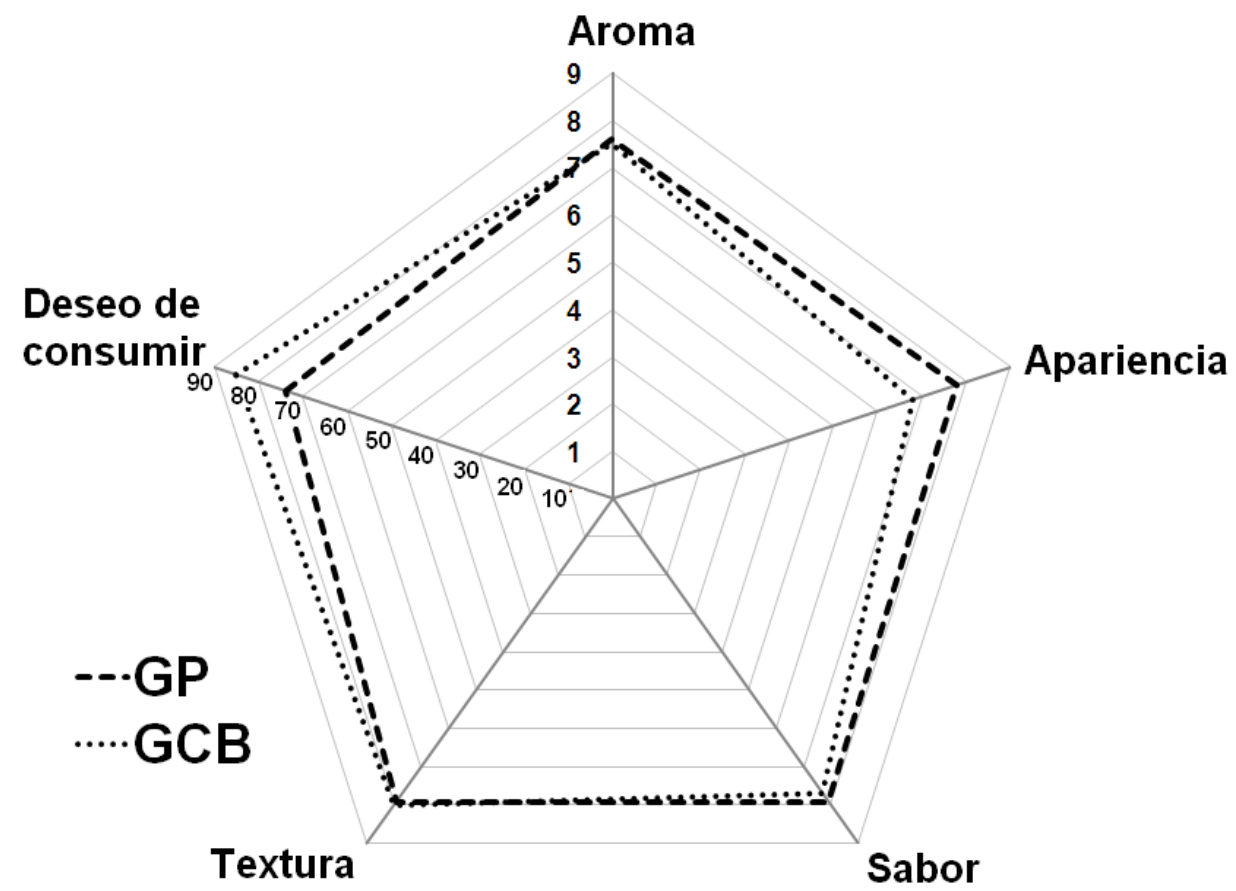

Fig 1: Distribución en escala hedónica, de los valores obtenidos a las cualidades sensoriales de las jaleas

Tabla 2: Evaluación de las cualidades sensoriales de las gelatinas de las patas de pollo. GUC = Gelatina Uva Comercial y GU = Gelatina Uva Experimental: d, e =Letras distintas indican diferencias significativas entre las muestras

\begin{tabular}{|l|c|c|c|c|c|}
\hline & \multicolumn{5}{|c|}{ Promedio de los Valores Sensoriales } \\
\hline Muestras & Aroma & Apariencia & Sabor & Textura & Deseo de consumir (\%) \\
\hline GUC & $8,1^{\mathrm{a}}$ & $8,3^{\mathrm{b}}$ & $8,4^{\mathrm{c}}$ & $7,9^{\mathrm{d}}$ & 90 \\
\hline GU & $7,7^{\mathrm{a}}$ & $7,5^{\mathrm{b}}$ & $7,0^{\mathrm{c}}$ & $6,4^{\mathrm{e}}$ & 83 \\
\hline
\end{tabular}

La Tabla 3 muestra la composición de las gelatinas experimental y comercial. La comparación de los valores de los componentes de la gelatina de las patas de pollo con gelatina comercial muestra que las cantidades de proteínas y de sodio en el primero caso es casi cuatro veces mayor que el segundo (Dr. Otker Brasil, 2010). Esto demuestra una calidad superior de la gelatina obtenida de las patas de pollo con respecto a la gelatina comercial. Por lo tanto, el consumo diario de una gelatina de las patas de pollo es equivalente al consumo de cuatro gelatinas comerciales, manteniendo las mismas propiedades nutricionales y medicinales (Lentz, 2008; Rivera et al., 2000). Así, los productos obtenidos tienen una buena calidad sensorial y química. También la materia prima tiene un precio de bajo y es más aceptable para los aspectos de la salud del ser humano que la obtenida de la piel de cerdo y ganado vacuno. Finalmente tiene una mayor aceptación por las comunidades judaica y el Islam (Gómes-Gulién et al., 2011; Karim y Bhat, 2009; MFRURAL, 2010). 


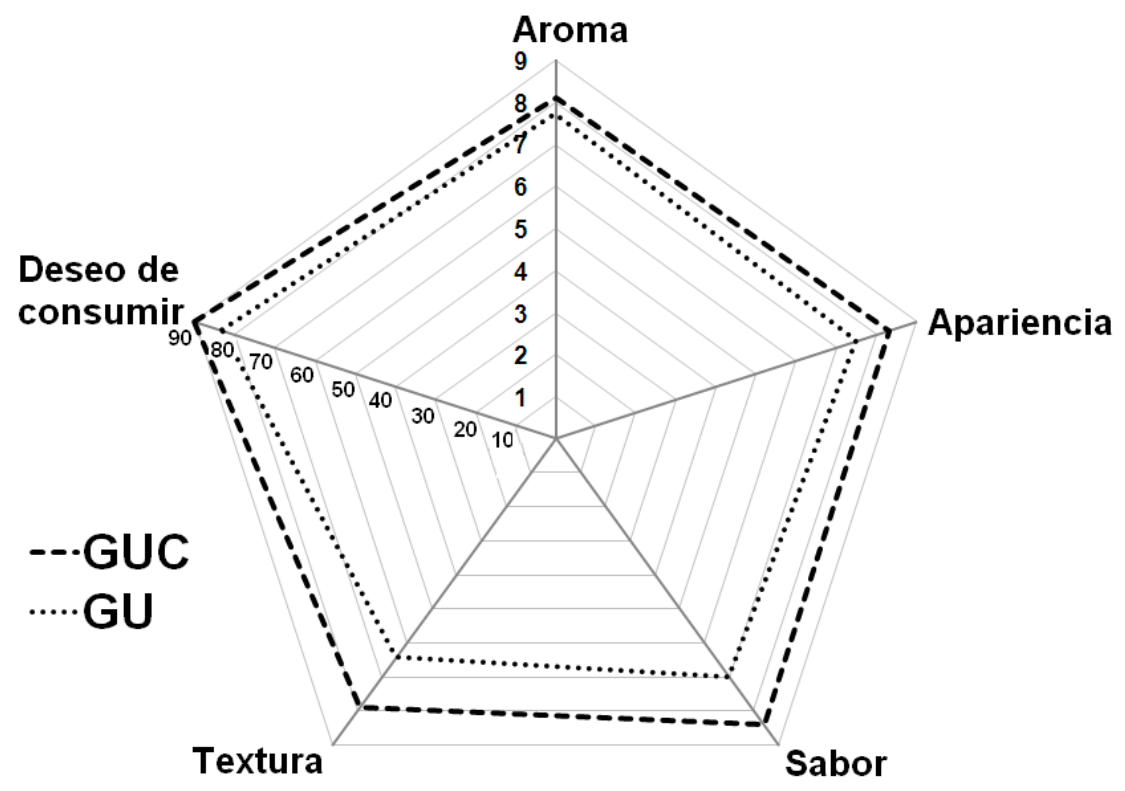

Fig 2: Distribución en la escala hedónica, de los valores obtenidos para las cualidades sensoriales de las gelatinas

Tabla 3: Composición química de las gelatinas de patas de pollos experimental y comercial, después de la preparación (Dr. Otker Brasil, 2010)

\begin{tabular}{|c|c|c|}
\hline \multirow{2}{*}{ Componentes } & \multicolumn{2}{|c|}{ Composición $(\mathrm{g} / \mathrm{10g})$} \\
\cline { 2 - 3 } & Experimental & Comercial \\
\hline Humedad & 6,4 & 8,5 \\
\hline & Base seca & $1,6^{\star}$ \\
\hline Proteínas $(\mathrm{g})$ & 6,30 & $66 \mathrm{mg}^{\star}$ \\
\hline Sodio $(\mathrm{g})$ & $247 \mathrm{mg}$ & \\
\hline
\end{tabular}

\section{CONCLUSIONES}

La mayoría de los catadores han aprobado los productos, mostrando un promedio cercano o por encima de la escala hedónica de 7 puntos, lo que indica una clasificación entre "gustado" y "gustó moderadamente", y más del $80 \%$ dijeron que consumirían los productos. Los niveles de proteínas en las jaleas y gelatinas de patas de pollo superaron en cuatro veces los niveles de los productos comerciales, lo que indica una superioridad y con respecto a la calidad química. Como el consumo de gelatina es indicado por hacer el bien para la salud humana, la dosis diaria de gelatina podría reducirse en cuatro veces al utilizar la gelatina de las patas de pollo, sin perder sus propiedades medicinales. Estos factores asociados con las cualidades químicas y sensoriales permiten la sustitución de las materias primas utilizadas actualmente por las patas de pollo.

El trabajo muestra cómo aprovechar de forma sustentable con la naturaleza, los residuos generados en la matanza de pollos y explorando el potencial de la industria del tarso de pollo en la obtención de un alimento de alta calidad, así como con buenas prácticas de producción. Este trabajo puede contribuir al desarrollo socioeconómico de regiones donde están instalados los frigoríficos y mataderos de aves. La inclusión de patas de pollo como un producto de valor añadido y no como residuo, podría generar nuevos puestos de trabajo en estas industrias y en empresas que procesan las jaleas y gelatinas. 


\section{AGRADECIMIENTOS}

La UNINOVE y el Programa PROSUP/CAPES por el apoyo económico.

\section{REFERENCIAS}

Alvis, A., H. S. Villada y D. C. Villada, Efecto de la temperatura y tiempo de fritura sobre las características sensoriales del ñame (Dioscorea alata), Información Tecnológica: 19(5), 19-26 (2008).

Arnesen, J. A. y A. Gildberg, Extraction and characterisation of gelatin from Atlantic salmon (Salmo salar) skin, Bioresource Technology: 98, 53-57 (2007).

Biazus, J. P. M., J. C. C. Santana y R. R. Souza, Modelagem empírica do processo de biodegradação de efluentes protéicos por enzimas de Carica papaya sp. Engenharia Agrícola e Ambiental: 10(2), 436-440 (2006).

Cheow, C. S., M. S. Norizah, Z. Y. Kyaw y N. K. Howell, Preparation and characterisation of gelatins from the skins of sin croaker (Johnius dussumieri) and shortfin scad (Decapterus macrosoma), Food Chemistry: 101, 386-391 (2007).

Coria, I.D. y Vegas, M., Determinación de la Efectividad de la remediación de suelos accidentalmente contaminados con ácido sulfúrico mediante métodos geofísicos, Información Tecnológica: 22(1), 3-8 (2011).

Costa, D. P. S., P. F. Romanelli y E. Trabuco, Aproveitamento de vísceras não comestíveis de aves para elaboração de farinha de carne, Ciência e Tecnologia de Alimentos: 28(3), 746-752 (2008).

Dr. Oetker Brasil, Produtos: Gelatinas. [online] Disponible en: <http://www.oetker.com.br/?actA=21 1\&linhalD=14 \&sublinhalD=19>, acceder a 20 de abril (2010).

Giménez, B., y otros 4 autores, Physico-chemical and film forming properties of giant squid (Dosidicus gigas) gelatin, Food Hydrocolloids: 23, 585-592 (2009).

Gómez-Guillén, M. C., B. Giménez, M. E. López-Caballero y P. Montero, Functional and bioactive properties of collagen and gelatin from alternative sources: A review, Food Hydrocolloids: 25, 1813-1827 (2011).

Hao, S., y otros 6 autores, The characteristics of gelatin extracted from sturgeon (Acipenser baeri) skin using various pretreatments, Food Chemistry: 115, 124-128 (2009).

Karim, A. A.; Bhat, R. Fish gelatin: properties, challenges, and prospects as an alternative to mammalian gelatins. Food Hydrocolloids 23, 563-576 (2009)

Lentz, K. A. Current methods for predicting human food effect - Mini-Review, The AAPS Journal: 10(2), 282-288 (2008).

Liu, D. C., Y. K. Lin y M. T. Chen, Optimum condition of extracting collagen from chicken feet and its characteristics. Asian-Australasian Journal of Animal Science: 14, 1638-1644 (2001).

MFRURAL - Classificados. Produtos rurais: preço dos pés de frango. Atualizado em 28/01/2010. [online] Disponível em <http://comprarvender.mfrural.com.br/detalhe.aspxcdp= 44686\&nmoca=procuro-compro-compro-pes-de-frango> Acessado em 22 de fevereiro de 2010.

Passarini, K. C., y otros 3 autores, Reutilización de las Aguas Residuales en la Irrigación de Plantas y en la Recuperación de los Suelos, Información Tecnológica, vol. 23(1) (2012). 
Rivera, J. A., J. G. Sebranek y R. E. Rust, Functional properties of meat by-products and mechanically separated chicken (MSC) in a high-moisture model petfood system. Meat Science: $55,61-66$ (2000).

Rodríguez, J. F. R. y L. Gualdron, Evaluación de la sustitución de grasa animal por grasa vegetal Insaturada en la Elaboración de un Embutido de Carne de Búfalo (Bubalus bubalis). Información Tecnológica: 22, 43-54 (2011).

Rodríguez, M. E., R. C. Miranda, R. Olivas y C. A. Sosa, Efectos de las condiciones de operación sobre la biosorción de $\mathrm{Pb2}+, \mathrm{Cd} 2+$ y Cr3+ en solución por Saccharomyces cerevisiae residual. Información Tecnológica: 19(6), 47-55 (2008).

Ronceros, B.A., J. I. Leiva, E. del C. Burgos y L. del C. Pardo, Efecto de la temperatura y tiempo de Almacenamiento sobre la Calidad del Tomate Deshidratado, Información Tecnológica: 19(5), (2008).

Santana, J. C. C., C. G. Dias, R. R. Souza y E. B. Tambourgi, Applying of neural network on the wine sensorial analysis from Barbados cherry, Journal of Food Process Engineering: 33, 365-378 (2010).

Santos, C.T., y otros 5 autores, Characterization and sensorial evaluation of cereal bars with jackfruit, Acta Scientiarum Technology: 33(1), 81-85 (2011).

Saxena, A., B. P. Tripathi, M. Kumar y V. K. Shahi, Membrane-based techniques for the separation and purification of proteins: An overview, Adv Colloid Interfac.: 145(1-2), 1-22 (2009).

Serna-Cock, L., M. Velásquez y A. A. Ayala, Efecto de la ultrafiltración sobre las propiedades reológicas de gelatina comestible de origen bovino, Información Tecnológica: 21, 91-102 (2010).

Silva, F. A. S., M. E. M. Duarte y M. E. R. M. Cavalcanti-Mata, New methodology for data interpretation of food sensorial analysis, Engenharia Agrícola: 30(5), 967-973 (2010).

Yan, H., Y. Wang, M. Jiang, J. H. Oh, J. Herring, J. y P. Zhou, 2-Step optimization of the extraction and subsequent physical properties of channel catfish (Ictalurus punctatus) skin gelatin. Journal of Food Science: 72(4), C188-C195 (2007). 